\title{
Neuropathological protocol for the study of unexplained stillbirth
}

\author{
Luca Roncati ${ }^{1}$, Francesco Piscioli ${ }^{2}$, Teresa Pusiol ${ }^{2}$, Anna M. Lavezzi ${ }^{3}$ \\ ${ }^{1}$ Department of Diagnostic and Clinical Medicine and of Public Health, Section of Pathology, University of Modena and Reggio \\ Emilia, Modena, ${ }^{2}$ Provincial Health Care Services, Institute of Pathology, Santa Maria del Carmine Hospital, Rovereto, \\ ${ }^{3}$ Department of Biomedical, Surgical and Dental Sciences, Lino Rossi Research Center, University of Milan, Milan, Italy
}

\begin{abstract}
An updated neuropathological protocol for the examination of the nervous system in case of unexplained stillbirth has been elaborated and presented in this review. It is focused on the examination of the nervous centers located in the brainstem, which are involved in monitoring the vital functions. Only through a deep analysis of the brainstem it is possible to highlight developmental alterations of these essential centers, and then provide a plausible explanation of the pathogenetic mechanism behind the death. The guidelines, drawn up on the basis of numerous researches performed by the authors, include a histopathological protocol, with an indication of standardized samples, and an immunohistochemical protocol for the study of biological markers, frequently involved in these deaths. The main risk factors that can be related to the neuronal alterations are also reported, together with the indications for the toxicological examination, which should be possibly applied. The authors hope that this protocol will be soon adopted in all the institutions where a fetal death, after a routine autopsy procedure, is diagnosed as "unexplained", in order to make standardized investigations on stillbirth. Nowadays, preventive strategies to decrease the incidence of these very distressing events for both parents and clinicians are necessary.
\end{abstract}

Key words: neuropathology, unexplained stillbirth, fetal autopsy, histology, immunohistochemistry, endocrine disrupting compounds.

\section{Introduction}

Inequality in use of fetal autopsy is poorly understood, despite the importance of autopsy in establishing the cause of stillbirth for future prevention [1]. Fetal deaths are in fact the most common adverse pregnancy outcomes in developed countries [4]. Despite a $35 \%$ decline in neonatal mortality over the last decades, thanks to public health campaigns promoting back sleep position, the stillbirth rate has not equally decreased [4]. Recently, the World Health Organization (WHO) has communicated that in 2015,
2.6 million stillbirths globally occurred, with more than 7178 deaths a day [4]. Obviously, parents want to know why their baby died in the womb and the chance of recurrence in future pregnancies. Therefore, the detection of the pathogenetic mechanism leading to stillbirth is very important, and not just to give an answer to parents, but above all to introduce preventive measures in order to lower prenatal mortality [1]. The value of a post-mortem examination, as the gold standard investigation following stillbirth, is well documented [18,19,23-26,29]. In particular, the impor-

\section{Communicating author}

Luca Roncati, MD, PhD, University of Modena and Reggio Emilia, Policlinico Hospital, 41124 Modena, Italy, phone: +390594224812,

fax: +390494224998, e-mail: emailmedical@gmail.com 
tance of an adequate fetal examination has been emphasized in a recent policy on the subject by the American Board of Pathology [3]. Nevertheless, the number of fetal autopsies performed worldwide not only has not increased, but it has even declined in the past years, when efforts to decrease stillbirth would just require policies to increase autopsy rates [1]. By contrast, in our unit, the "Lino Rossi" Research Center of Milan University, thanks to Italian law no. 31 "Regulations for diagnostic post mortem investigation in victims of sudden infant death syndrome (SIDS) and unexpected fetal death", there has been a trend for increasing the number of stillbirth autopsy examinations since 2006 [2]. Precisely, this law states that all fetuses, which suddenly died without any apparent cause after 25 weeks of gestation, must be submitted to a diagnostic post-mortem investigation, and that the findings of the in-depth autopsy, the data about pregnancy, fetal development and delivery, and the information related to risk factors, must be collected by the medical personnel (obstetricians, gynecologists, pathologists, etc.) and recorded in the National Data Bank. Researchers can then draw on all the collected results in order to make reliable investigations on the etiology of stillbirth, and improve preventative strategies for these very distressing events for both parents and clinicians. The "Lino Rossi" Research Center has developed an appropriate investigative post-mortem protocol that includes, specifically, the in-depth examination of the autonomic nervous system. The application of this protocol has proved to be greatly useful when a routine fetal autopsy did not show a possible cause of death. It is well known, in fact, that, despite the identification and classification of possible causes of stillborn infants into maternal, fetal, placental and related to external factors, sometimes also combined together, the death remains unexplained in a percentage between 33\% and 50\% of cases [6]. However, a diagnostic orientation can be obtained from the careful examination, performed by an experienced neuropathologist, of the brain centres checking the vital functions, mostly sited in the brainstem. Detailed examinations of these structures performed in our Institution for several decades, even thanks to Italian law no. 31, in a cohort of over 500 unexplained stillbirths, highlighted, in the great part of cases, their subtle developmental morphological and/or functional alterations, frequently related to environmental risk factors, such maternal smoking. The findings provide insight into the specific patho- physiological process leading to prenatal death. Here, we describe the neuropathologic procedure applied in our Center and which should be adopted in all the anatomopathological institutions where a fetal death, after a standard autopsy procedure, is diagnosed as "unexplained stillbirth" or, more appropriately, "sudden intrauterine unexplained death syndrome" (SIUDS) [20].

\section{Preamble}

Only the intact fetal brain (i.e. not macerated or liquefied) can be submitted to the protocol presented below. Then, in case of fetal death, the first assessment consists in the exclusion of cases with brain autolysis. Our previous studies have identified, in well-preserved fetal brains, the main brainstem nuclei and structures that should be examined, hitherto highlighted only in experimental animals. Given the impossibility to perform experiments in humans, the homologous human nuclei have been identified on the basis of morphological criteria of similarity with regard to the location, the cytoarchitecture and number of neurons and applying, when possible, immunohistochemical methods able to highlight the same neurotransmitters expressed in specific nervous structures in rats. Through this original methodology, it was possible to recognize, in particular, the Kölliker-Fuse nucleus, the facial/parafacial complex, the pre-Bötzinger nucleus in the pons/medulla oblongata, and the intermediolateral nucleus in the rostral spinal cord, up to now unidentified in humans $[10,12,13,16]$. These nervous centers, as demonstrated in experimental studies, are linked together via inter-neuronal synapses into the brainstem in a "respiratory network", able to modulate one another to maintain a regular breathing activity. It should be pointed that, while death related to improper breathing control is easily comprehensible as a possible cause of SIDS, it is difficult to understand how ventilatory alterations can lead to death during intrauterine life, when breathing is not yet a vital condition. We propose, as explanation, that in the last weeks of pregnancy, advancing towards the time of birth, a general check of all the neuronal centers essential for extra-uterine life takes place [17]. Sudden unexpected prenatal deaths could therefore be ascribed to a selective process of self-suppression in the presence of developmental alterations particularly of the respiratory centers, preventing an even more serious and stressful event of a neonatal death [11]. 


\section{Histopathological protocol}

Figure 1 shows a simplified methodology for the brainstem's examination, consisting in the sampling of three specimens (Fig. 1). The first specimen, pontomesencephalic, includes the upper third of the pons and the adjacent portion of caudal mesencephalon. The second extends from the upper part of the medulla oblongata to the adjacent caudal portion of the pons. The third specimen encloses the obex. A fourth sample is taken from the rostral tract of the spinal cord (Fig. 1). Transverse serial sections of each sample are made at intervals of $60 \mu \mathrm{m}$. For each level, from eight to ten 5 - $\mu$ m-thick sections are obtained, two of which are stained using hematoxylin-eosin and Klüver-Barrera for histological examination, while the remaining sections are treated, according to the needs, with specific immunohistochemical techniques in order to evaluate the expression of functional markers (as reported in the following paragraph). Any remaining sections are saved for further investigations and stained as deemed necessary. In Figure 2, a schematic representation of the typical histological sections obtained from the four specimens is reported, with the indication of the main nuclei and structures that can be examined (Fig. 2). Overall, they are: the hypoglossus, dorsal motor vagal, tractus solitarius, ambiguus, infe- rior olivary, pre-Bötzinger, arcuate, dorsal and ventral cochlear, medial inferior vestibular, obscurus and pallidus raphé nuclei in the medulla oblongata; the locus coeruleus, facial/parafacial complex, retrotrapezoid, superior olivary complex, superior and lateral vestibular, Kölliker-Fuse, median and magnus raphé nuclei in the pons; the inferior colliculus, substantia nigra, dorsal and caudal linear raphé nuclei in the caudal mesencephalon. In the spinal cord, the intermedio-lateral nucleus is well recognizable. A diagnosis of "hypoplasia" of a given nucleus is formulated when it shows a significantly decreased number of neurons and/or a decreased area in transverse histological sections, compared to the mean values obtained in age-matched controls. Four of the aforementioned structures, the Kölliker-Fuse nucleus, facial/parafacial complex, pre-Bötzinger nucleus and the intermediolateral nucleus, components of the "respiratory network", are in particular of great interest, and must be always examined in SIUDS. They are entirely enclosed in the four samples taken (Fig. 3). These centers, all components of the respiratory network, are able to coordinate each other through excitatory and/or inhibitory connections, in relation to the need, to control the breathing activity before and after birth. Their evaluation can be speeded by examining a few selected

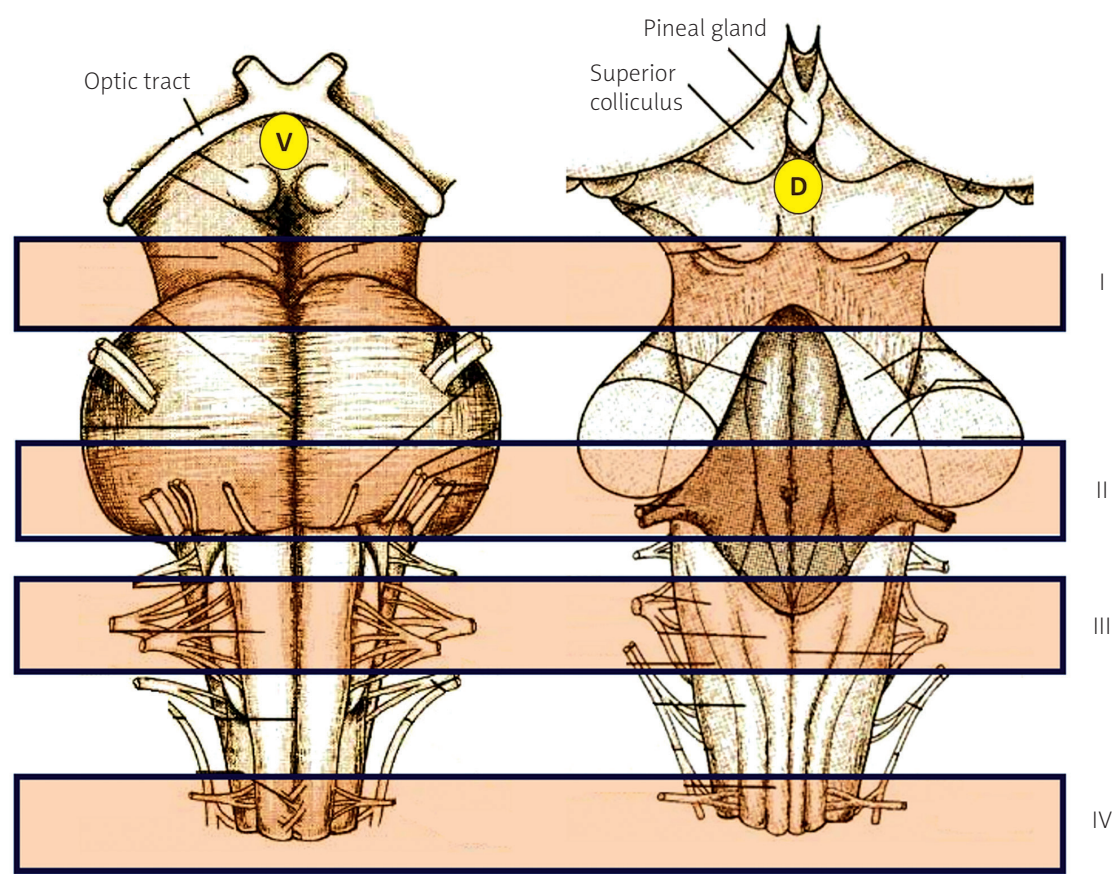

Fig. 1. Schematic representation of the brainstem and spinal cord sampling in four (I, II, III, IV) transversal planes in the ventral (V) and dorsal (D) view. 


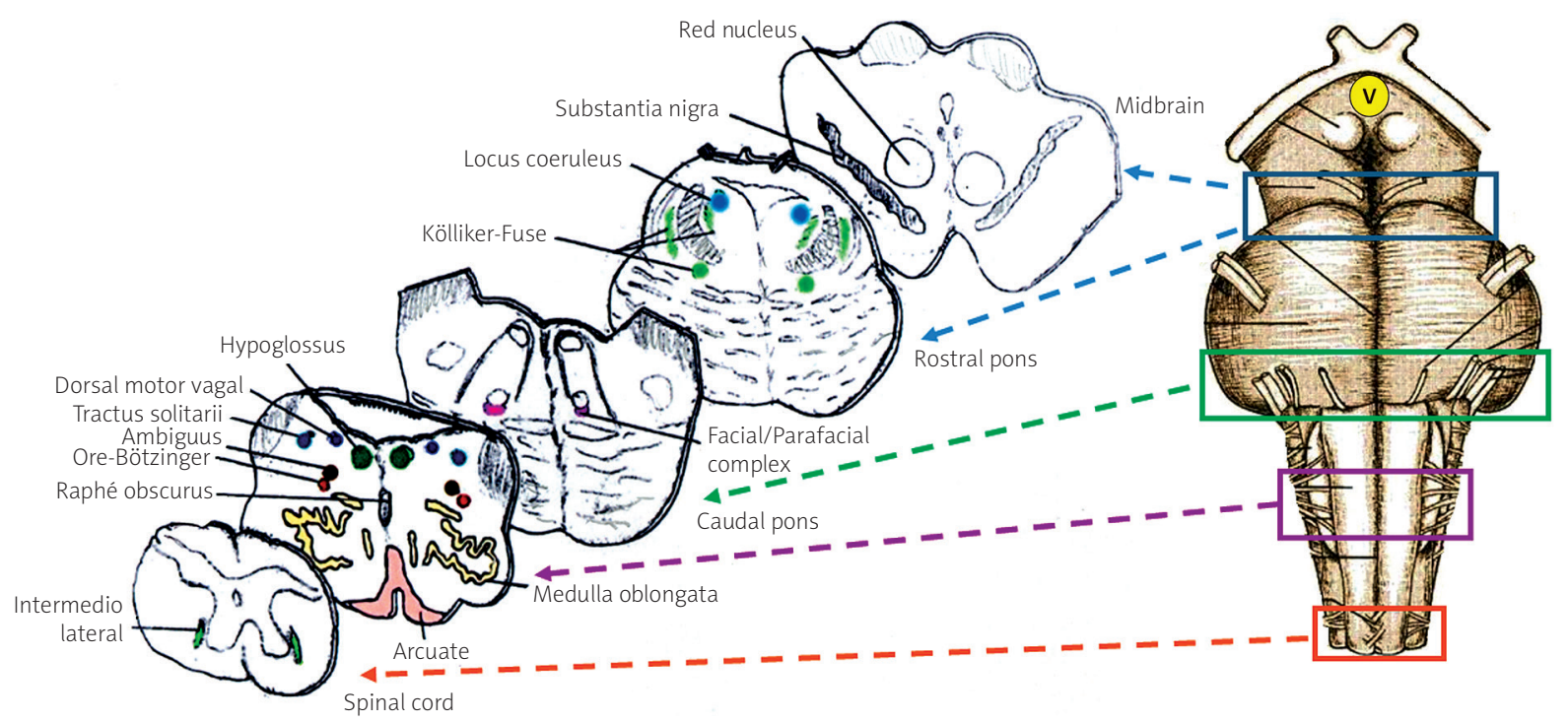

Fig. 2. Schematic representation of the histological sections obtainable from the sampling illustrated in Figure 1, with an indication of the main nuclei and examinable structures.

histological sections. The corresponding specimens are easily recognizable on the basis of precise landmarks that are the presence of the superior cerebellar peduncle decussation in the rostral pons (for the Köl-

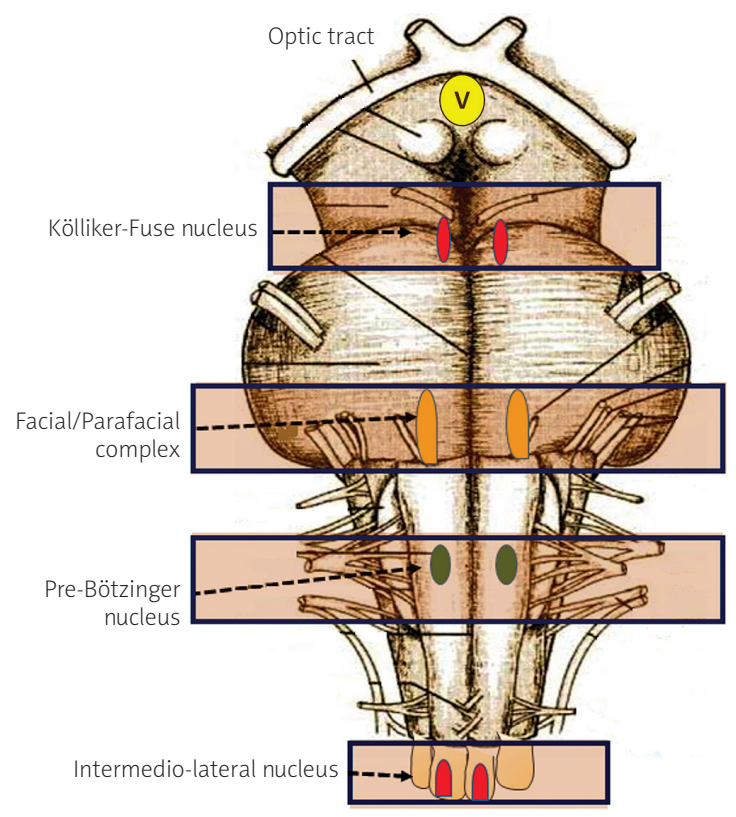

Fig. 3. Schematic representation of the localization of the main respiratory centers (Kölliker-Fuse nucleus, facial/parafacial complex, pre-Bötzinger nucleus and intermedio-lateral nucleus): they are totally included in the four samples obtained.
liker-Fuse nucleus analysis), of the medial nucleus of the superior olivary complex in the caudal pons (for the facial/parafacial complex), of the dorsal accessory of the inferior olivary nucleus in the medulla oblongata (for the pre-Bötzinger nucleus) and of dorsal and ventral horns, adjacent to the central canal, in the rostral spinal cord (for the intermediolateral nucleus).

\section{Immunohistochemical protocol}

The reserved sections for the immunohistochemical analyses are treated according to standard procedures in order to evaluate functional parameters, whose altered expression can represent a very significant finding in SIUDS. These biological markers are: neuronal nuclear antigen (NeuN), nicotinic acetylcholine (nACh) receptors (Rs), serotonin (5-HT) Rs, somatostatin (SS) Rs, apoptosis and gliosis.

\section{NeuN}

This antigen is strongly expressed in the nucleus of post-mitotic healthy neurons already in fetal life, with the inexplicable exception of several neuronal cell types (precisely, Purkinje cells and dentate nucleus neurons in the cerebellum, neurons of the inferior olivary nucleus in the medulla oblongata, and all the glial cells). By excluding these neurons, a decreased NeuN immunopositivity or absolute negativity can be indicative of neuronal degeneration. In particular, immunoreactivity is significantly weakened after a severe injury, such as 
cerebral hypoxia/ischemia [33]. Then, it is very important to apply this technique in all SIUDS to obtain useful information about the physiological general status of neurons. The related detailed immunohistochemical technique is available in our previous article [9].

\section{nACh Rs}

The $n A C h R s$ regulate critical aspects of brain maturation during the intrauterine life, through neurochemical transmission of acetylcholine among the neurons. These receptors are ion channels in the cytoplasmic membrane, consisting of a different combination of $\alpha$ and $\beta$ subunits. To date, a total of $10 \alpha$ and $4 \beta$ different subunits have been described [17]. The nAChRs can be opened not only by the neurotransmitter acetylcholine, but also by nicotine (hence the adjective "nicotinic"). Fetal brain damage caused by maternal smoking in pregnancy mainly results from the interaction of nicotine with nAChRs. We specifically suggest addressing the study on the $\alpha 7-n A C h R$ subtype, given its important involvement in neuronal differentiation, synaptogenesis and formation of brain circuits in fetal life and its demonstrated high vulnerability to toxic effects of nicotine [7].

\section{5-HT Rs}

5-HT is a neuromodulator transmitter above all involved in neurotrophic processes during brain development, deputy to the control of neural circuits, including the respiratory network in the ponto-medullary brainstem. It is produced by the serotonergic neurons of the raphé system, a complex of nuclei spread along the midline of the brainstem. In detail, they are the caudal linear raphé nucleus, the dorsal raphé nucleus, the median raphé nucleus, the raphé magnus nucleus, the raphé obscurus nucleus and the raphé pallidus nucleus. A decrease in 5-HT Rs in medullary regions containing raphé neurons has been described by us in SIUDS [8].

\section{SS Rs}

SS is a neuropeptide with a wide distribution in the central nervous system. During fetal development, in particular, SS-containing neurons are prevalently concentrated in the brainstem nuclei involved in the ventilatory regulation, as demonstrated by the high immunopositivity of their receptors [5]. This suggests that SS is normally deputy to the inhibition of breathing in utero, while the detection of SS-im- munonegativity leads to presume a fatal intrauterine respiratory activity. For this reason, it is definitely recommended to study this transmitter in SIUDS; the related method can be retrieved from our previous works [14,15].

\section{Apoptosis and gliosis}

The immunostaining for the detection of apoptosis (TUNEL method) and glial fibrillary acid protein (GFAP) are also recommended to obtain further information about a possible abnormal increase of neuronal death, over the physiological levels, and the presence of reactive gliosis, a well-known marker of neurodegenerative processes $[19,21]$.

\section{Toxicological protocol}

It is important, for each case, to collect a complete clinical history, with particular reference to the maternal lifestyle, including information related to the main potential risk factors (such as maternal smoking, alcohol, drug abuse and air pollution).

\section{Nicotine}

Among the risk factors, maternal smoking during pregnancy is worldwide recognized as highly associated to fetal death. The mothers must be asked for information about their smoking habit before and during pregnancy. Their reply should be possibly validated through a toxicological research in fetal hair of cotinine, the main metabolite of nicotine, characterized by a long half-life and great stability [32]. The protocol generally includes a qualitative cotinine test to detect the presence or absence of the substance in the hair. A quantitative testing by liquid chromatography-mass spectrometry (LC-MS) system can also be applied to evaluate the cotinine concentration [32].

\section{Pesticides}

Recently, our original investigations have demonstrated the adverse effects of persistent pollutants, such as pesticides and household insecticides, on brain development [30,31]. Exactly as nicotine, these harmful chemicals belong to the category of the "endocrine disrupting compounds" (EDCs) $[27,28]$. Traces of highly toxic substances, as organochlorine and organophosphate pesticides ( $\alpha$ and $\gamma$-chlordane, chlorfenvinphos, chlorpyrifos, p,p-DDT, p,p-DDE, endrin, $\alpha$ - and $\beta$-endosulfans), 
have been directly detected in the brain of fetuses, died in agricultural areas where pesticides are used [22].

\section{Conclusions}

The post-mortem examination, in case of intrauterine death, is worldwide recognized as important for determining the cause of stillbirth and directing management of subsequent pregnancy [1]. It is necessary, however, to point out that the lack of uniform post-mortem protocols applied in the different centers has hindered significant studies in this field. Furthermore, in the routine fetal autopsy, the examination of the nervous system has received up to now poor attention. Here, we have proposed a neuropathological protocol for a concise and specific examination of the nervous system, and in particular of the brainstem, very useful when the routine autopsy does not reveal a precise cause of fetal death. The application of these guidelines, drawn up after many years of studies in this field, frequently allow to highlight developmental morphological and/or physiological neuronal alterations that could explain the pathogenesis of SIUDS, considering also their correlation with the exposure to environmental risk factors, such as EDCs. The core objective of our work has been to provide, in the simplest way, the steps for the accurate neuropathological examination of the brainstem. The protocol is the culmination of our pluriannual scientific experience in this field. We strongly hope that the examination of the central nervous system, following this protocol, becomes a specialized component of the fetal autopsy, above all when a clear cause of death is not found. The application of this procedure is furthermore the best way to collect a rich body of information about all possible neurological alterations in the fetal brain underlying SIUDS, in order to reduce its incidence and mitigate the surrounding social concern. Pathologists are then invited to adopt this protocol, making it a standardized universal procedure in unexplained stillbirths.

\section{Disclosure}

Authors report no conflict of interest.

\section{References}

1. Auger N, Tiandrazana RC, Healy-Profitós J, Costopoulos A. Inequality in fetal autopsy in Canada. J Health Care Poor Underserved 2016; 27: 1384-1396.
2. Available at: http://users.unimi.it/centrolinorossi/files/gazz ufficiale.pdf

3. Available at: http://www.abpath.org/FetalAutopsyPolicy.pdf

4. Available at: http://www.who.int/maternal_child_adolescent/ epidemiology/stillbirth/en/

5. Chigr F, Najimi M, Leduque P, Charnay Y, Jordan D, Chayville JA, Tohyama M, Kopp N. Anatomical distribution of somatostatin immunoreactivity in the infant brainstem. Neuroscience 1989; 29: 615-628.

6. Fretts RC. Etiology and prevention of stillbirth. Am J Obstet Gynecol 2005; 193: 1923-1935.

7. Lavezzi AM, Cappiello A, Pusiol T, Corna MF, Termopoli V, Matturri L. Pesticide exposure during pregnancy, like nicotine, affects the brainstem $\alpha 7$ nicotinic acetylcholine receptor expression, increasing the risk of sudden unexplained perinatal death. J Neurol Sci 2015; 348: 94-100.

8. Lavezzi AM, Casale V, Oneda R, Weese-Mayer DE, Matturri L. Sudden infant death syndrome and sudden intrauterine unexplained death: correlation between hypoplasia of raphé nuclei and serotonin transporter gene promoter polymorphism. Pediatr Res 2009; 66: 22-27.

9. Lavezzi AM, Corna MF, Matturri L, Neuronal nuclear antigen (NeuN): a useful marker of neuronal immaturity in sudden unexplained perinatal death. J Neurol Sci 2013; 329: 45-50.

10. Lavezzi AM, Corna MF, Mehboob R, Matturri L. Neuropathology of the intermediolateral nucleus of the spinal cord in sudden unexplained perinatal and infant death. Int I Dev Neurosci 2010; 28: 133-138.

11. Lavezzi AM, Ferrero S, Matturri L, Roncati L, Pusiol T. Developmental neuropathology of brainstem respiratory centers in unexplained stillbirth: What's the meaning? Int J Dev Neurosci 2016; 53: 99-106.

12. Lavezzi AM, Matturri L. Functional neuroanatomy of the human pre-Bötzinger complex with particular reference to sudden unexplained perinatal and infant death. Neuropathology 2008; 28: 10-16.

13. Lavezzi AM, Matturri L. Hypoplasia of the parafacial/facial complex: a very frequent finding in sudden unexplained fetal death. Open Neurosci I 2008; 2: 1-5.

14. Lavezzi AM, Ottaviani G, Matturri L. Involvement of somatostatin in breathing control before and after birth, and in perinatal and infant sudden unexplained death. Folia Neuropathol 2004; 42: 59-65.

15. Lavezzi AM, Ottaviani G, Matturri L. Role of somatostatin and apoptosis in breathing control in sudden perinatal and infant unexplained death. Clin Neuropathol 2004; 23: 304-310.

16. Lavezzi AM, Ottaviani G, Rossi L, Matturri L. Cytoarchitectural organization of the parabrachial//ölliker-Fuse complex in man. Brain Dev 2004; 26: 316-320.

17. Lindstrom J, Anand R, Gerzanich V, Peng X, Wang F, Wells G. Structure and function of neuronal nicotinic acetylcholine receptors. Prog Brain Res 1996; 109: 125-137.

18. Manganaro L, Scialpi M, Piscioli F, Pusiol T, Roncati L. MRI prenatal diagnosis of genitourinary abnormalities in a case of inconclusive ultrasonography. J Obstet Gynaecol 2016; 36: 762-763.

19. Manganaro L, Vinci V, Giancotti A, Gerli S, Cozzi DA, Pusiol T, Scialpi M, Roncati L. Bi-parametric magnetic resonance imaging applied to obstetrics. J Obstet Gynaecol 2017; 37: 670-672. 
20. Martin LJ. Neuronal cell death in nervous system development, disease and injury. Int J Mol Med 2001; 7: 455-478.

21. Matturri L, Pusiol T, Lavezzi AM. Proposal of the acronym "SIUDS" for unexplained stillbirths, like "SIDS". J Neonatal Biol 2014; 3: 5-6.

22. Norenberg MD. Astrocytes responses to CNS injury. I Neuropathol Exp Neurol 1994; 53: 213-220.

23. Pusiol T, Lavezzi A, Matturri L, Termopoli V, Cappiello A, Piscioli F Roncati L. Impact assessment of endocrine disruptors on sudden intrauterine and infant death syndromes. Eur J Forensic Sci 2016; 3: 8-15

24. Pusiol T, Lavezzi AM, Piscioli F, Roncati L. Sudden infant death due to imported malignant malaria in Europe. Eur J Forensic Sci 2015; 2: 18-20

25. Pusiol T. Roncati L, Lavezzi AM, Taddei F, Piscioli F, Ottaviani G. Sudden fetal death due to dualism of the sino-atrial node. Cardiovasc Pathol 2016; 25: 325-328.

26. Roncati L, Barbolini G, Fano RA, Rivasi F. Fatal Aspergillus flavus infection in a neonate. Fetal Pediatr Pathol 2010; 29: 239-244.

27. Roncati L, Barbolini G, Pusiol T, Piscioli F, Maiorana A. New advances on placental hydrops and related villous lymphatics. Lymphology 2015; 48: 28-37.

28. Roncati L, Piscioli F, Pusiol T. The endocrine disrupting chemicals as possible stillbirth contributors. Am J Obstet Gynecol 2016; 215: 532-533.

29. Roncati L, Piscioli F, Pusiol T. The endocrine disruptors among the environmental risk factors for stillbirth. Sci Total Environ 2016; 563-564: 1086-1087.

30. Roncati L, Pusiol T, Piscioli F, Barbolini G, Maiorana A, Lavezzi A. The first 5-year-long survey on intrauterine unexplained sudden deaths from the Northeast Italy. Fetal Pediatr Pathol 2016; 35: 315-326.

31. Roncati L, Pusiol T, Piscioli F, Lavezzi AM. Neurodevelopmental disorders and pesticide exposure: the northeastern Italian experience. Arch Toxicol 2016; 91: 603-604.

32. Roncati L, Termopoli V, Pusiol T. Negative role of the environmental endocrine disruptors in the human neurodevelopment Front Neurol 2016; 7: 143.

33. Tzatzarakis MN, Vardavas Cl, Terzi I, Kavalakis M, Kokkinakis M Liesivuori J, Tsatsakis AM. Hair nicotine/cotinine concentrations as a method of monitoring exposure to tobacco smoke among infants and adults. Hum Exp Toxicol 2012; 31: 258-265.

34. Unal-Cevik I, Kilinç M, Gürsoy-Ozdemir Y, Gurer G, Dalkara T. Loss of NeuN immunoreactivity after cerebral ischemia does not indicate neuronal cell loss: a cautionary note. Brain Res 2004; 1015: 169-174. 